\title{
Molecular Environments of Supernova Remnants $\dagger$
}

\author{
Yang Chen ${ }^{1,2}$, Bing Jiang ${ }^{1}$, Ping Zhou ${ }^{1}$, Yang $\mathbf{S u}^{3}$, Xin Zhou ${ }^{3,2}$, \\ Hui $\mathbf{L i}^{4,1}$, and Xiao Zhang ${ }^{1}$ \\ ${ }^{1}$ Department of Astronomy, Nanjing University, Nanjing 210093, China \\ email: ygchen@nju.edu.cn, bjiang@nju.edu.cn, pingzhou@nju.edu.cn, zxmysky@163.com \\ ${ }^{2}$ Key Laboratory of Modern Astronomy and Astrophysics, Nanjing University, Ministry of \\ Education, Nanjing 210093, China \\ ${ }^{3}$ Purple Mountain Observatory, 2 West Beijing Road, Nanjing 210008, China \\ email: yangsu@pmo.ac.cn, xinzhou@pmo.ac.cn \\ ${ }^{4}$ Department of Astronomy, University of Michigan, 500 Church Street, Ann Arbor, \\ MI 48109, USA \\ email: hliastro@umich.edu
}

\begin{abstract}
There are about 70 Galactic supernova remnants (SNRs) that are now confirmed or suggested to be in physical contact with molecular clouds (MCs) with six kinds of evidence of multiwavelength observations. Recent detailed CO-line spectroscopic mappings of a series of SNRs reveal them to be in cavities of molecular gas, implying the roles the progenitors may have played. We predict a linear correlation between the wind bubble sizes of main-sequence OB stars in a molecular environment and the stellar masses and discuss its implication for supernova progenitors. The molecular environments of SNRs can serve as a good probe for the $\gamma$-rays arising from the hadronic interaction of the accelerated protons, and this paper also discusses the $\gamma$-ray emission from MCs illuminated by diffusive protons that escape from SNR shocks.
\end{abstract}

Keywords. ISM: supernova remnants, ISM: clouds, ISM: bubbles, ISM: molecules, gamma-rays: ISM, stars: early-type

\section{Introduction}

The interplay between supernova remnants (SNRs) and their molecular environments plays an important role in many aspects of astrophysical studies. Molecular gas takes up about half mass of Galactic interstellar medium, and most core-collapse supernovae are believed to be located close to giant molecular clouds (MCs) where their progenitor stars are given birth to (e.g., Huang \& Thaddeus 1986). The SNR shock waves propagating in molecular environments can compress, heat, excite, ionize, and even dissociate molecules. They are suggested to be one of the mechanisms of triggering star formation. They influence the chemical evolution of the gas and produce otherwise impossible or seldom detected molecular emission (e.g., $1720 \mathrm{MHz} \mathrm{OH}$ maser, $\mathrm{HCO}^{+}$, etc.). Shock interaction with molecular clouds can generate $\gamma$-rays as a result of neutral pion decay after p-p collision (hadronic interaction), which may serve as a probe of SNR shock acceleration of relativistic protons. Once an SNR-MC association is established, the kinematic distance of the SNR can be determined with the local standard of rest (LSR) velocity of the MC.

$\dagger$ Supported by the 973 Program grant 2009CB824800, the NSFC grants 11233001, 11103082, and 11203013, grants 20120091110048 and 20110091120001 from the Educational Ministry of China, and grant 2011M500963 from the China Postdoctoral Science Foundation. 
This article is composed of contents on SNR-MC associations, some of our CO-line observations, bubbles in molecular environments, and probe for hadronic interaction.

\section{Census of SNR-MC associations}

How many among the $\gtrsim 300$ Galactic SNRs are in physical contact with MCs? There have been some endeavors in surveying and cataloging the SNR-MC associations. In an early CO-line survey toward 26 outer $\left(l=70^{\circ}-210^{\circ}\right)$ Galactic SNRs, about half were found spatially coincident with large MC complexes (Huang \& Thaddeus 1986). After a survey of $1720 \mathrm{MHz} O \mathrm{OH}$ maser emission towards Galactic SNRs, Frail et al. (1996) and Green et al. (1997) revealed the unique diagnostic relation between such masers and the SNR shocks in MCs and identified nearly a score of SNRs interacting with MCs. Seta et al. (1998) listed 26 SNRs detected in $\mathrm{CO}$ - and $\mathrm{H}_{2}$-line emissions along the line of sight, but no physical evidence of shock-MC interaction was given for them.

Recently, we presented a catalog of the interacting SNRs, with a summary of six kinds of multiwavelength observational evidence for judging the contact of SNRs with MCs (Jiang et al. 2010). Among the six observational evidences, firstly, the $1720 \mathrm{MHz} \mathrm{OH}$ maser is the most important, which is presently widely accepted as a signpost of SNR shock-MC interaction. When the molecular gas is compressed by a C-type shock to a density of order $\sim 10^{5} \mathrm{~cm}^{-3}$ and the temperature reaches $50-125 \mathrm{~K}$, collisional pumping causes the population inversion of the hyperfine rotational level ${ }^{2} \Pi_{3 / 2}(J=5 / 2)$ of $\mathrm{OH}$ molecules, from which the $1720 \mathrm{MHz}$ maser arises (Lockett et al. 1999). Since this OH satellite line was noted by Goss \& Robinson (1968), explorations and surveys in the line have been repeatedly made toward Galactic SNRs, and the masers have been detected from 25 SNRs. Secondarily, molecular $\left(\mathrm{CO}, \mathrm{HCO}^{+}, \mathrm{CS}\right.$, etc.) line broadening or asymmetric profile is another important kinematic evidence of shock perturbation of the molecular gas (e.g., DeNoyer 1979). Line broadening is present in 18 SNRs listed in our table. The third kind of evidence is high high-to-low excitation line ratio in line wings (Seta et al. 1998). In the broad wings of the shocked ${ }^{12} \mathrm{CO}$, where both the $J=2-1$ and $J=1-0$ emissions are optically thin, their ratio can be $\gtrsim 1$. The fourth is the detection of near-infrared (IR) emission, e.g., [Fe II] line or ro-vibrational lines of $\mathrm{H}_{2}$ (such as $\mathrm{H}_{2} 1-0 \mathrm{~S}(1)$ line at $2.12 \mu \mathrm{m}$ and $\mathrm{H}_{2} 0-0 \mathrm{~S}(0)-\mathrm{S}(7)$ lines), due to shock excitation. The fifth is the specific IR colors suggesting molecular shocks, e.g., $3.6 \mu \mathrm{m} / 5.8 \mu \mathrm{m}$ and $4.5 \mu \mathrm{m} / 8 \mu \mathrm{m}$ in the Spitzer IRAC observation (see Reach et al. 2006 and Fig.2 therein). The sixth is the morphological agreement or correspondence of molecular features with SNR features (e.g., arc, shell, interface, etc.). Besides the $\mathrm{OH}$ masers, a combination of the sixth (spatial) evidence with one of the other four kinematic/physical evidences is also regarded as convincing criteria for judging the SNR-MC interaction. Thus, in our catalog, there are 34 SNRs "confirmed", 11 "probable", and 19 "possible" to be in physical contact with MCs. A most recent CO survey for $l=60^{\circ}-190^{\circ}$ showed additional six SNRs with their radio morphology in a good spatial relation with MCs, without direct evidence for the interaction (Jeong et al. 2012), thus raising the number of "possible" interacting SNRs to 25.

\section{Our CO observations of a series of SNRs}

The $\mathrm{OH}$ satellite line masers have proven to be a powerful tool for the identification of interacting SNRs, nonetheless they cannot be used to investigate the detailed information of molecular environments of the SNRs. Also, a number of SNR-MC associations may be elusive in the search in the maser line because of the weak emission below the detection 
thresholds. For this purpose, CO, other than $\mathrm{H}_{2}$ (without a permanent electric dipole moment), is a practical tracer of MCs and commonly used for case studies of SNR$\mathrm{MC}$ interaction. Here we review our recent $\mathrm{CO}$ observations of a series of interacting SNRs made with the millimeter and sub-millimeter telescopes in the Purple Mountain Observatory at Delingha, Seoul Radio Astronomy Observatory, Koelner Observatory for Submillimeter Astronomy, and other observatories.

\subsection{Kes 69}

Kes 69 is morphologically characterized by the bright radio, IR, and X-ray emissions only at the southeastern boundary. The $1720 \mathrm{MHz} O \mathrm{OH}$ masers are detected in both the northeast and the southeast, but with different LSR velocities (Green et al. 1997; Hewitt et al. 2008). Our ${ }^{12} \mathrm{CO}(J=1-0)$ observation (Zhou et al. 2009) discovers a molecular arc in the LSR velocity interval $77-86 \mathrm{~km} \mathrm{~s}^{-1}$ (consistent with $85 \mathrm{~km} \mathrm{~s}^{-1}$ for the southeastern maser). The arc is in good morphological agreement with the multiwavelength partial shell of the SNR. The $\mathrm{HCO}^{+}$emission is detected at the position of the radio brightness peak also at the LSR velocity $V_{\mathrm{LSR}}=85 \mathrm{~km} \mathrm{~s}^{-1}$, which is consistent with the presence of the southeastern maser, both resulting from C-shock interaction. These evidences strongly suggest that Kes 69 is physically associated with the MC at the systemic velocity $85 \mathrm{~km} \mathrm{~s}^{-1}$. From this velocity, a kinematic distance $5.2 \mathrm{kpc}$ is derived. We ascribe the multiwavelength emissions arising from the southeastern partial shell of the SNR to the impact of the SNR shock on a dense, clumpy patch of molecular gas, which is likely to be the cooled debris of the material swept up by the progenitor's stellar wind.

\subsection{Kes 75}

The young composite SNR Kes 75, with a pulsar wind nebula inside, displays only a half shell in the south. We find that the ${ }^{12} \mathrm{CO}(J=1-0)$ line profile of the $V_{\mathrm{LSR}}=45^{-}$ $58 \mathrm{~km} \mathrm{~s}^{-1} \mathrm{MC}$ is broadened in the blue wing and a molecular shell unveiled in this wing (45-51 $\mathrm{km} \mathrm{s}^{-1}$ ) encloses a cavity ( $\mathrm{Su}$ et al. 2009). The southern part of the molecular shell is in good morphological agreement with the SNR's half shell shown in X-rays, mid$\mathrm{IR}$, and radio continuum. These spatial and kinematic evidences indicate that Kes 75 is physically associated with the MC at the systemic velocity $54 \mathrm{~km} \mathrm{~s}^{-1}$. The associated large cloud has a mass $\gtrsim 10^{4} M_{\odot}$. The distance to this SNR is thus determined to be $10.6 \mathrm{kpc}$. The presence of the dense molecular shell is interpreted to be due to the same reason as that of the molecular arc in Kes 69 .

\subsection{Kes 78}

Kes 78 is a shell-type SNR, but only the eastern half is radio bright. There is a $86 \mathrm{~km} \mathrm{~s}^{-1}$ $\mathrm{OH}$ maser at the eastern boundary (Koralesky et al. 1998). The unidentified TeV source J1852-000 to the east of the remnant is detected by the HESS $\gamma$-ray telescopet, which implies that this SNR may have a relation with the very high energy emission. Our CO line observations toward this SNR find that it is interacting with the MC complex at the systemic velocity $V_{\mathrm{LSR}}=81 \mathrm{~km} \mathrm{~s}^{-1}$ (Zhou \& Chen 2011). The ${ }^{13} \mathrm{CO}$ emission shows the presence of a dense cloud to the east, from which the maser may arise and which also seems crudely correspondent to the location of the extended $\mathrm{TeV}$ source. The strong ${ }^{12} \mathrm{CO}$ emission in general spatially corresponds to the eastern bright radio shell, and the spatial extent of the SNR is consistent with a ${ }^{12} \mathrm{CO}$ cavity. ${ }^{12} \mathrm{CO}$ lines are found to be broadened in some boundary regions including the eastern maser region, and ${ }^{12} \mathrm{CO}$ $J=2-1 / J=1-0$ ratio is generally elevated along the SNR boundary. These are all the

$\dagger$ http://www.mpi-hd.mpg.de/hfm/HESS/pages/home/som/2011/02/ 
kinematic signatures of shock perturbation in the molecular gas. The distance to Kes 78 is estimated to be $4.8 \mathrm{kpc}$ based on the systemic LSR velocity. An XMM-Newton X-ray spectral analysis for the northeastern boundary infers an age $6 \mathrm{kyr}$ for the remnant.

\section{4. $3 C 396$}

Analogous to Kes 75, 3C 396 is another composite SNR containing a pulsar wind nebula and is semi-circular-shaped, too. We find that the western edge, bright in radio, IR, and X-rays, is perfectly confined by a molecular wall (of mass $\sim 10^{4} M_{\odot}$ ) revealed in ${ }^{12} \mathrm{CO}(J=1-0)$ line at $V_{\mathrm{LSR}} \sim 84 \mathrm{~km} \mathrm{~s}^{-1}$ (Su et al. 2011). The $\mathrm{CO}$ emission gradually gets faint from west to east, which is indicative that the eastern region is of low gas density. Noticeably, a finger/pillar-like molecular structure (of mass $\sim 4 \times 10^{3} M_{\odot}$ ) in the southwest intrudes into SNR edge. The pillar, which may have been shocked at the tip, should be the reason why the X-ray and radio emissions get brightened at the southwestern boundary and some IR filaments are present there. The evidences for the SNR-MC interaction also include the relatively elevated ${ }^{12} \mathrm{CO} J=2-1 / J=1-0$ line ratios in the southwestern pillar tip and the molecular patch at the northwestern boundary, as well as the redshifted ${ }^{12} \mathrm{CO}(J=1-0$ and $J=2-1)$ wings $\left(86-90 \mathrm{~km} \mathrm{~s}^{-1}\right)$ of an eastern $V_{\mathrm{LSR}} \sim 81 \mathrm{~km} \mathrm{~s}^{-1}$ molecular patch. The X-ray analysis of the hot gas infers an age $\sim 3 \mathrm{kyr}$ for the remnant and the derived relative abundances of $\mathrm{Si}, \mathrm{S}$, and $\mathrm{Ca}$ of the ejecta are consistent with a B1-B2 progenitor star.

\section{5. $3 C 397$}

3C 397 is a thermal composite (or mixed-morphology) SNR with an abnormal rectangular shape. Our ${ }^{12} \mathrm{CO}(J=1-0)$ observation shows that the remnant is confined in a molecular gas cavity and embedded at the edge of a giant $\mathrm{MC}$ at the systemic velocity $V_{\mathrm{LSR}}=$ $32 \mathrm{~km} \mathrm{~s}^{-1}$ (Jiang et al. 2010). The column density of the environmental molecular gas has a gradient that increases from the southeast to the northwest and is perpendicular to the Galactic plane, in agreement with the elongation direction of the remnant. Solid evidence for the SNR-MC interaction is provided by the ${ }^{12} \mathrm{CO}$ line broadening of the $\sim 32 \mathrm{~km} \mathrm{~s}^{-1}$ component that is detected at the westmost boundary of the remnant, which is consistent with the impact of the Fe rich ejecta in the region (Jiang \& Chen 2010). The systemic velocity of the molecular gas places 3C 397 at a kinematic distance of $10.3 \mathrm{kpc}$. The mean ambient molecular density is $\sim 10-30 \mathrm{~cm}^{-3}$, which can explain the high volume emission measure of the X-ray emitting gas. For the westmost line-broadened region, the density of the disturbed molecular gas is deduced to be of order $10^{4} \mathrm{~cm}^{-3}$ based on the the pressure balance between the cloud shock and the X-ray emitting hot gas and can be ascribed to very dense clumps, implying a multi-phase gas environment there.

\subsection{Kes 79 and $W 49 B$}

SNRs Kes 79 and W 49B have also been observed and preliminarily show interesting molecular environments.

Kes 79, harboring a central compact object, was demonstrated by the Chandra observation to have multi-shell structure, which implies a complicated surrounding environment (Sun et al. 2004). Our CO observation finds that this remnant is spatially coincident with a molecular cavity at $V_{\mathrm{LSR}} \sim 113 \mathrm{~km} \mathrm{~s}^{-1}$ (Fig. 1 ). The ${ }^{12} \mathrm{CO} J=2-1 / J=1-0$ ratio is also found to be elevated, as high as $\gtrsim 1$, in some boundary regions.

$\mathrm{W} 49 \mathrm{~B}$, sorted as a thermal composite, has an incomplete radio shell with centrally brightened thermal X-rays. A barrel-shaped structure with coaxial rings was revealed in the $1.64 \mu \mathrm{m}$ [Fe II] image, and a strip of $2.12 \mu \mathrm{m}$ shocked $\mathrm{H}_{2}$ emission extends outside of the $[\mathrm{Fe}$ II] emission to the southeast; the X-ray jet-like structure is along the axis of 

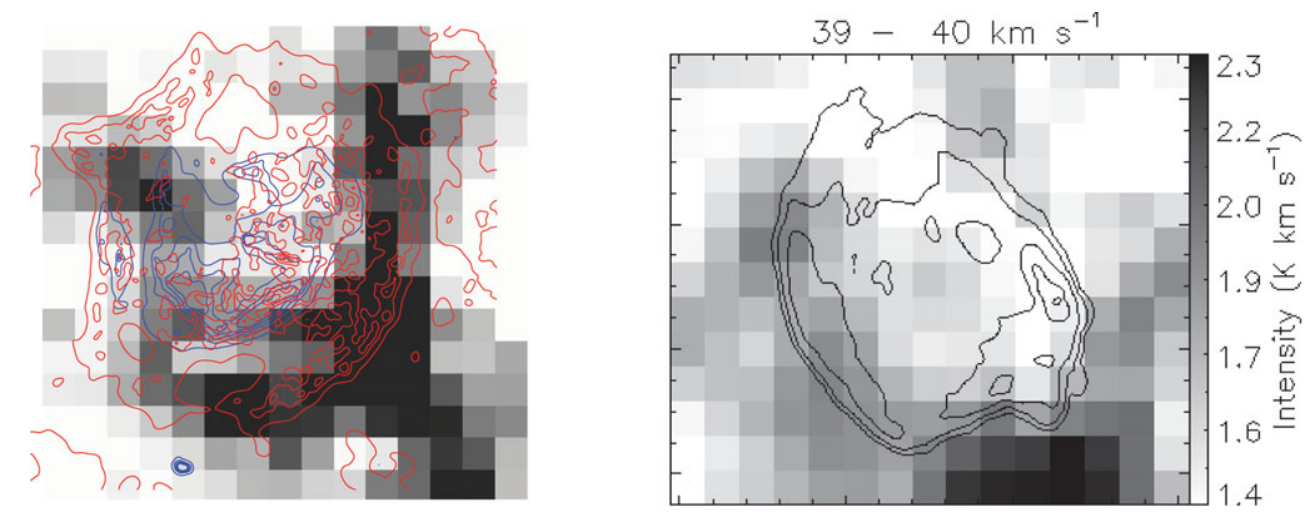

Figure 1. Left: ${ }^{12} \mathrm{CO}(J=2-1)$ emission map at $V_{\mathrm{LSR}}=112-115 \mathrm{~km} \mathrm{~s}^{-1}$ for Kes 79 , overlaid with radio continuum contours (red) and X-ray contours (blue). The size of each grid cell is 1 arcmin. Right: ${ }^{12} \mathrm{CO}(J=2-1)$ emission map at $V_{\mathrm{LSR}}=39-40 \mathrm{~km} \mathrm{~s}^{-1}$ for $\mathrm{W} 49 \mathrm{~B}$, overlaid with radio continuum contours. The size of each grid cell is 0.5 arcmin.

the barrel (Keohane et al. 2007). It has been suggested that W 49B originated inside a wind-blown bubble interior to a dense MC. By CO observation, we have noticed that this SNR seems to be coincident with a molecular cavity at $V_{\mathrm{LSR}} \sim 39 \mathrm{~km} \mathrm{~s}^{-1}$ (Fig. 1), but no robust kinematic evidence has been obtained yet. If this SNR-MC association is true, the kinematic distance to $\mathrm{W} 49 \mathrm{~B}$ is $9.3 \mathrm{kpc}$.

The above observations show a common phenomenon that these interacting SNRs all evolve in molecular cavities/bubbles, which may be created by their progenitors' activities (i.e., most probably, stellar winds). The cavities/bubbles are very unlikely to be produced by the SNR shocks, because the supernova explosion energy would otherwise be much higher than the canonical budget $10^{51} \mathrm{erg}$.

\section{Bubbles in molecular environment}

A bubble blown by a main sequence star achieves the maximum size when the bubble is in pressure equilibrium with the ambient medium (Chevalier 1999). Chen et al. (2013) show a linear relation for the bubble size $R_{\mathrm{b}}$ for main-sequence OB stars: $p^{1 / 3} R_{\mathrm{b}} \propto M$, where $p$ is the pressure of the surrounding interclump medium and $M$ the stellar mass. Actually, by inserting parameters that are observationally determined and model-estimated into Chevalier's (1999) formula, they find that, for 15 exemplified main-sequence OB stars, $p^{1 / 3} R_{\mathrm{b}}$ does correlate linearly with $M$, and a good regression for the relation can be obtained as

$$
p_{5}^{1 / 3} R_{\mathrm{b}}=\alpha\left(M / M_{\odot}\right)-\beta \mathrm{pc}
$$

where $p_{5} \equiv(p / k) /\left(10^{5} \mathrm{~cm}^{-3} \mathrm{~K}\right), \alpha=1.21 \pm 0.05$, and $\beta=8.98 \pm 1.76$.

In a giant MC, the mean pressure is $p_{5} \sim 1$ (Krumholz et al. 2009), and this number is indeed needed to confine the dense clumps and supports the cloud against gravitational collapse (Blitz 1993; Chevalier 1999).

Eq. (4.1) provides a powerful way to assess the initial masses of the progenitors for SNRs associated with molecular clouds. For an initial mass below $25-30 M_{\odot}$, the stars will terminate their lives in Type II-P supernova (SN) explosion, which takes up the greatest majority of SNe II, after the red supergiant (RSG) phase, without further launching WolfRayet winds. The post-main sequence stellar winds reach an extent much smaller than the main-sequence bubbles. The SN shocks will rapidly pass through the circumstellar 
Table 1. Galactic SNRs with molecular shells/in molecular cavities

\begin{tabular}{|c|c|c|c|c|}
\hline $\mathbf{S N R}^{\mathrm{ref}}$ & \multicolumn{4}{|c|}{$\mid$ Distance $[\mathbf{k p c}]\left|R_{\mathrm{b}}[\mathbf{p c}]\right| M\left[M_{\odot}\right] \mid$} \\
\hline G21.8-0.6 (Kes 69) ${ }^{1}$ & 1 & 6.2 & 13 & $\sim 18$ \\
\hline G29.7-0.3 (Kes 75$)^{2}$ & 1 & 10.6 & I & $\sim 12$ \\
\hline G32.8-0.1 (Kes 78$)^{3}$ & $\mid$ & 4.8 & 17 & $\sim 21$ \\
\hline G33.6+0.1 $(\text { Kes } 79)^{4}$ & 1 & 7 & I & $\sim 14$ \\
\hline G39.2-0.3 (3C 396 $)^{5}$ & $\mid$ & 6.2 & I & $\sim 13$ \\
\hline G41.1-0.3 (3C 397 $)^{6}$ & $\mid$ & 10.3 & $4.5-7$ & $\sim 12$ \\
\hline $\mathrm{G} 43.3-0.2(\mathrm{~W} 49 \mathrm{~B})^{7}(?)$ & $\mid$ & 9.3 & I & $\sim 13$ \\
\hline G54.4-0.3( $\mathrm{HC} 40)^{8}$ & $\mid$ & $3 / 7$ & $18 / 43$ & $\sim 22 / ?$ \\
\hline G263.9-3.3 (Vela) $)^{9,19}(?)$ & | & 0.29 & | 14-19 & $\sim 21$ \\
\hline G347.3-0.5 (RX J1713-3946) $)^{11,12}$ & & 1.1 & I & $\sim 15$ \\
\hline
\end{tabular}

Notes: The "?" symbol means that the SNR-MC association needs to be confirmed.

References-1: Zhou et al. (2009); 2: Su et al. (2009); 3: Zhou \& Chen (2011); 4: Sun et al. (2004) 5: Su et al. (2011); 6: Jiang \& Chen(2010); 7: this paper 8: Junkes et al. (1992); 9: Moriguchi et al. (2001); 10: Dodson et al. (2003); 11: Fukui et al. (2003); 12: Moriguchi et al. (2005)

material and impact the massive shells of the bubbles, with drastic deceleration (Chen et al. 2003). Thus, such SNRs reflect mostly the bubble sizes, and the sizes can be used to infer the progenitors' masses.

Table 1 lists the interacting SNRs that are known or suggested to have molecular shells or be in molecular cavities, which include the Vela SNR and RX J1713-3946 in addition to those described in $\S 3$. The Vela SNR coincides with a molecular void at a velocity range of $V_{\mathrm{LSR}}=-5$ to $85 \mathrm{~km} \mathrm{~s}^{-1}$ (Moriguchi et al. 2001). It is suggested that the molecular clumps are pre-existent, rather than having been swept up by the SNR shock, and that the SNR may have been expanding in a low density medium. RX J1713.7-3946 appears to be confined in a molecular gas cavity at $V_{\mathrm{LSR}} \sim-11$ to $-3 \mathrm{~km} \mathrm{~s}^{-1}$ (Fukui et al. 2003; Moriguchi et al. 2005). It is suggested that this SNR is still in the free expansion phase and the non-decelerated blast wave is colliding with the dense molecular gas after it traveled in a low-density cavity that perhaps was produced by the stellar wind or pre-existing supernovae (Fukui et al. 2003).

The estimates of progenitors' masses for the SNRs are given in the last column, on the assumption that the interclump pressure to be a constant: $p_{5} \approx 1$. Some of the estimates can be compared with other available independent assessments, which show considerably good consistency. For 3C 396, Su et al. (2011), based on Chandra observation, derived a progenitor mass of $13-15 M_{\odot}$ from the metal abundances of the X-ray-emitting gas that may be dominated by the SN ejecta. For 3C 397, a recent XMM-Newton X-ray study has analyzed the metal abundances of the SN ejecta and thus assessed the progenitor's mass to be $11-15 M_{\odot}$ (Safi-Harb et al. in preparation). For the Vela SNR, an SN II-P progenitor mass was suggested to be $15-20 M_{\odot}$ based on the moderate size of the wind-blown bubble (Gvaramadze 1999).

\section{Probe for hadronic interaction}

The origin of cosmic rays (CRs) has drawn more and more attention motivated by the recent decades' $\mathrm{X}$ - and $\gamma$-ray observations. It is generally believed that the CRs below the "knee" $\left(3 \times 10^{15} \mathrm{eV}\right)$ are of Galactic origin. The candidate sites where the Galactic CRs 
are accelerated include SNRs, binaries, superbubbles, Galactic center, etc., and SNRs are usually regarded as the most important. Relativistic electrons accelerated up to an energy $10^{13}-10^{14} \mathrm{eV}$ by the SNR shocks are evidenced by the X-ray synchrotron emission arising from them. Yet, conclusive evidence for the acceleration of relativistic protons remains poor. An indirect way is to detect the $\gamma$-ray emission generated by the hadronic interaction of the accelerated protons, namely, neutral pion decay after the collision of the protons with the baryons of the environmental dense gas. Thus, the MCs with which SNRs are associated or interacting are a perfect probe for the hadronic process.

In the second Fermi-LAT GeV source catalog, there are 89 sources whose $95 \%$ confidence error ranges overlap with SNRs, and among which $45 \%$ are of chance coincidences but six correspond to firmly identified $\gamma$-ray emitting SNRs (Nolan et al. 2012). Among the TeV sources, about ten correspond to SNRs (Holder 2012). Also, a correlation has been proposed between a class of $\mathrm{GeV}-\mathrm{TeV} \gamma$-ray sources that are coincident with interacting SNRs and the $1720 \mathrm{MHz} \mathrm{OH}$ masers (Hewitt et al. 2009). However, it is not easy to differentiate whether the $\gamma$-rays arising from the SNRs are hadronic or leptonic emission. Recent years, a progress has been made by analysing the "illuminating" effect of the nearby MCs by the SNR shock-accelerated protons (e.g., Aharonian \& Atoyan 1996; Gabici et al. 2009; Li \& Chen 2010, 2012; Ohira et al. 2011).

Among these work, Li \& Chen $(2010,2012)$ established an "accumulative diffusion" model for the escaping protons and naturally interpreted the $\mathrm{GeV}-\mathrm{TeV}$ spectra of nine interacting SNRs. W28 is one of the prototype interacting SNRs, in contact with a large $\mathrm{MC}$ in the north and harboring a large amount of $1720 \mathrm{MHz}$ masers (Frail et al. 1994). Four $\mathrm{TeV}$ sources in the area are detected by the H.E.S.S. $\gamma$-ray telescope, positionally coincident with the northern large MC and three small MCs in the south (Aharonian et al. 2008). The three southern clouds seem to be outside the reach of the W28 blast wave, and Fermi-LAT detected no significant GeV emission for two of them (Abdo et al. 2010). They show that the various relative $\mathrm{GeV}-\mathrm{TeV}$ brightness of the four $\gamma$-ray sources results from the hadronic process of the accelerated protons that escape from the shock front and bombard the nearby MCs at different radii. The "illuminating" protons are considered to be an accumulation of the diffusive protons escaping from the shock front throughout the history of the SNR expansion. For the various distances of the MCs from the SNR center, the resulting proton spectra can have prominently different shapes. Adopting different centric distances, the $\gamma$-ray spectral fit for the four sources well explains their $\mathrm{GeV}-\mathrm{TeV}$ spectral properties. It is also implied that the spectral index 2.7 at the high energy side is caused by diffusion other than directly by acceleration, and the spectral break (from 2.1 to 2.7 ) at around $1 \mathrm{GeV}$ can naturally appear due to the accumulative diffusion effect.

This model, with improvement by incorporating the finite volume of molecular clouds, is further applied to nine $\gamma$-ray emitting interacting SNRs (W 28, W 41, W 44, W 49B, W 51C, Cygnus Loop, IC 443, CTB 37A and G349.7+0.2). Like that aforementioned, the $\sim \mathrm{GeV}$ spectral breaks are commonly present in these SNRs; and most of them have a spectral $\left(E^{2} d F / d E\right)$ "platform" extending from the break to lower energies. This refined model perfectly explains the $\sim \mathrm{GeV}$ spectral breaks and the "platforms", together with the available $\mathrm{TeV}$ data. It is also found that the index of diffusion coefficient $\delta$ derived from the spectral fit is in the range $0.5-0.7$, analogous to the Galactic average, and the diffusion coefficient for the CRs around the SNRs $\left(\chi \sim 10^{-2}\right)$ is essentially two orders of magnitude lower than the Galactic average, which is indicative of the suppression of CR diffusion near SNRs.

$\gamma$-rays from molecular clouds illuminated by diffusive protons that escape from interacting SNRs strongly support the scenario that hadrons accelerated by SNR shocks contribute to the Galactic CRs. A discovery of the spectral rising below $\sim 200 \mathrm{MeV}$ for 
IC 443 and W44 as an unique pion-decay signature is newly released after this Symposium (Ackermann et al. 2013).

So far, nearly a dozen of SNRs are proposed to emit hadronic $\gamma$-rays, including the historical SNRs Cas A and Tycho. Against the previous suggestion for tenuous medium surrounding Tycho responsible for the hadronic emission, it is argued that the $\gamma$-rays are produced from dense ambient matter, most probably MCs, too (see Zhang et al. 2013).

\section{References}

Abdo, A. A., Ackermann, M., Ajello, M., et al. 2010, ApJ, 718, 348

Ackermann, M., Ajello, M., Allafort, A., et al. 2013, Science, 339, 807

Aharonian, F., Akhperjanian, A. G., Bazer-Bachi, A. R., et al. 2008, A\& A, 481, 401

Aharonian, F. A. \& Atoyan, A. M. 1996, A\&A, 309, 917

Blitz, L. 1993, in: E. H. Levy \& J. I. Lunine (eds.), Protostars and Planets III (Tucson: Univ. of Arizona), 125

Chen, Y., Zhang, F., Williams, R. M., \& Wang, Q. D. 2003, ApJ, 595, 227

Chen, Y., Zhou, P., \& Chu, Y.-H. 2013, ApJ(Letters), in press, arXiv:1304.5126

Chevalier, R. A. 1999, ApJ, 511, 798

DeNoyer, L. K. 1979, ApJ(Letters), 232, L165

Dodson, R., Legge, D., Reynolds, J. E., \& McCulloch, P. M. 2003, ApJ, 596, 1137

Frail, D. A., Goss, W. M., Reynoso, E. M., et al. 1996, AJ, 111, 1651

Frail, D. A., Goss, W. M., \& Slysh, V. I. 1994, ApJ(Letters), 424, L111

Fukui, Y., Moriguchi, Y., Tamura, K., et al. 2003, PASJ, 55, L61

Gabici, S., Aharonian, F. A., \& Casanova, S. 2009, MNRAS, 396, 1629

Goss, W. M. \& Robinson, B. J. 1968, ApL, 2, 81

Green, A. J., Frail, D. A., Goss, W. M., \& Otrupcek, R. 1997, AJ, 114, 2058

Gvaramadze, V. 1999, A\& A, 352, 712

Hewitt, J. W., Yusef-Zadeh, F., \& Wardle, M. 2008, ApJ, 683, 189

Hewitt, J. W., Yusef-Zadeh, F., \& Wardle, M. 2009, ApJ(Letters), 706, L270

Holder, J. 2012, Astroparticle Physics, 39, 61

Huang, Y.-L. \& Thaddeus, P. 1986, ApJ, 309, 804

Jeong, I.-G., Byun, D.-Y., Koo, B.-C., et al. 2012, ApSS, 342, 389

Jiang, B., Chen, Y., Wang, J., et al. 2010, ApJ, 712, 1147

Jiang, B. \& Chen, Y. 2010, Sci. China G: Phys. Astron., 53, 267

Junkes, N., Fuerst, E., \& Reich, W. 1992, A\&AS, 96, 1

Keohane, J. W., Reach, W. T., Rho, J., \& Jarrett, T. H. 2007, ApJ, 654, 938

Koralesky, B., Frail, D. A., Goss, W. M., Claussen, M. J., \& Green, A. J. 1998, AJ, 116, 1323

Krumholz, M. R., McKee, C. F., \& Tumlinson, J. 2009, ApJ, 699, 850

Li, H. \& Chen, Y. 2010, MNRAS(Letters), 409, L35

Li, H. \& Chen, Y. 2012, MNRAS, 421, 935

Lockett, P., Gauthier, E., \& Elitzur, M. 1999, ApJ, 511, 235

Moriguchi, Y., Yamaguchi, N., Onishi, T., Mizuno, A., \& Fukui, Y. 2001, PASJ, 53, 1025

Moriguchi, Y., Tamura, K., Tawara, Y., Sasago, H., Yamaoka, K., Onishi, T., \& Fukui, Y. 2005, ApJ, 631, 947

Nolan, P. L., Abdo, A. A., Ackermann, M., et al. 2012, ApJS, 199, 31

Ohira, Y., Murase, K., \& Yamazaki, R. 2011, MNRAS, 410, 1577

Reach, W. T., Rho, J., Tappe, A., et al. 2006, AJ, 131, 1479

Seta, M., Hasegawa, T., Dame, T. M., et al. 1998, ApJ, 505, 286

Su, Y., Chen, Y., \& Yang, J., et al. 2009, ApJ, 694, 376

$\mathrm{Su}$, Y., Chen, Y., \& Yang, J., et al. 2011, ApJ, 727, 43

Sun, M., Seward, F. D., Smith, R. K., \& Slane, P. O. 2004, ApJ, 605, 742

Zhang, X., Chen, Y., Li, H., \& Zhou, X. 2013, MNRAS(Letters), 429, L25

Zhou, P. \& Chen, Y. 2011, ApJ, 743, 4

Zhou, X., Chen, Y., Su, Y., \& Yang, J. 2009, ApJ, 691, 516 